\title{
Mamdani Fuzzy Systems for Modelling and Simulation: A Critical Assessment
}

\author{
Segismundo S. Izquierdo' and Luis R. Izquierdo ${ }^{2}$ \\ ${ }^{1}$ Department of Industrial Organization, University of Valladolid, Paseo del Cauce 59, 47011 Valladolid, Spain \\ ${ }^{2}$ University of Burgos, Edificio la Milanera, C/Villadiego s/n, 09001 Burgos, Spain \\ Correspondence should be addressed tosegis@eii.uva.es \\ Journal of Artificial Societies and Social Simulation 21(3) 2, 2018 \\ Doi: 10.18564/jasss.3444 Url: http://jasss.soc.surrey.ac.uk/21/3/2.html \\ Received: 14-11-2016 Accepted: 28-03-2018Ｐublished: 30-06-2018
}

\begin{abstract}
Fuzzy logic presents many potential applications for modelling and simulation. In particular, this paper analyses one of the most popular fuzzy logic techniques: Mamdani systems. Mamdani systems can look particularly appealing because they are designed to incorporate expert knowledge in the form of IF-THEN rules expressed in natural language. While this is an attractive feature for modelling and simulating social and other complex systems, its actual application presents important caveats. This paper studies the potential use of Mamdani systems to explore the logical consequences of a model based on IF-THEN rules via simulation. We show that in the best-case scenario a Mamdani system provides a function that complies with its generating set of IF-THEN rules, which is a different exercise from that of finding the relation or consequences implied by those rules. In general, the logical consequences of a set of rules cannot be captured by a single function. Furthermore, the consequences of an IF-THEN rule in a Mamdani system can be very different from the consequences of that same rule in a system governed by the most basic principles of logical deductive inference. Thus, care must be taken when applying this tool to study "the consequences" of a set of hypothesis. Previous analyses have typically focused on particular steps of the Mamdani process, while here we present a holistic assessment of this technique for (deductive) simulation purposes.
\end{abstract}

Keywords: Social Simulation, Decision Support Systems, Deductive Inference, Fuzzy Logic, Mamdani

\section{Introduction}

1.1 Fuzzy logic can be traced to Lofti Zadeh's 1965 seminal paper titled "Fuzzy Sets" (Zadeh 1965). Since then, the theory of fuzzy sets has been successfully applied in a wide range of fields, but - contrary to its creator's initial expectations - most applications are found outside the boundaries of the Social Sciences. In Zadeh's own words: "When I wrote my 1965 paper, I expected that fuzzy set theory would be applied primarily in the realm of human sciences. Contrary to my expectation, fuzzy set theory and fuzzy logic are applied in the main in physical and engineering sciences" (Arfi 2010, foreword).

1.2 The use of fuzzy set theory in the Social Sciences is certainly not widespread, but ever since the early pioneering proposals (see e.g. Cioffi-Revilla 1981, Smithson 2012) there has been a constant interest in potential applications of fuzzy logic for social modelling and simulation (Arfi 2010, Ragin \& Pennings 2005). Nowadays one can find several noteworthy examples in the field of social simulation and related domains (Denize et al. 2012, Dykstra et al. 2015: Fleischmann 2005, Fort \& Pèrez 2005; Góngora y Moreno \& Gutierrez-Garcia|2017:| Hassan et al. 2007, 2010, 2011; Lee et al. 2013, 2014; Olaru \& Smith 2005| Sabater et al.|2006), and various proposals have been made to apply fuzzy logic in different social contexts (see e.g. Power 2009; Neumann et al.|2011). There is also a NetLogo (Wilensky 1999) extension developed to facilitate the implementation of fuzzy approaches in agent-based simulations (Izquierdo et al.|2015), already being used in social simulation studies (Anand et al. 2017). More generally, a wide range of computer tools have been developed over the past years to make use of fuzzy logic in modelling, simulation and decision making, and many general computing environments such as MatLab implement popular fuzzy methods, like the so-called "Mamdani fuzzy inference" (MATLAB 2016).

1.3 This widespread availability of ready-to-use software, the willingness of a community eager to apply new tools to the analysis of complex systems, and the apparent simplicity of the basic principles of fuzzy logic have led 
to a situation in which any scholar can nowadays build and use a simple fuzzy logic model in a matter of hours. This observation naturally constitutes a positive advancement in many aspects, but it also means that there is a potential risk of misapplying the theory and/or misusing the tools.

1.4 This paper focuses on the use of Mamdani inference, which is one of the most popular techniques in applied fuzzy logic. Mamdani inference is mainly used in fuzzy control (Driankov et al. 1996), but has also been applied in various other fields such as management and finance (Bojadziev 2007)|Bodenhofer et al.|2012), economics Stojić 2012: West \& Linster 2003), risk assessment (Darbra et al. 2008: Nait-Said et al. 2008), and even for measuring the quality of education (Valdés-Pasarón et al.|2011).

1.5 In this paper we assess the usefulness of Mamdani systems for modelling, simulation and decision making. To achieve this end, we put together and synthesise several results already known in the field of fuzzy logic, and illustrate them with examples purposefully designed for our objectives. Our assessment is intended to be accessible to researchers who may not be experts in the field of fuzzy logic but are interested in exploring the possibilities of this particular fuzzy technique, with a focus on social simulation.

1.6 Mamdani systems have proven to be a very useful tool for function approximation and control (Cao et al. 2001) - an aspect that does offer interesting potential applications in the social sciences too-, so we will not discuss that aspect here; in this paper we analyse their potential usefulness as a tool for logical deductive inference to study, via simulation, the consequences and behaviour of a model defined by means of IF-THEN rules.

1.7 Mamdani systems are most often classified as a form of Approximate Reasoning, which has been defined as "the process or processes by which a possible imprecise conclusion is deduced from a collection of imprecise premises" (Pal \& Mandal|1991). This categorization, together with the fact that the core component of a Mamdani system is a set of IF-THEN rules, can easily mislead one to believe that Mamdani systems can provide the logical implications of the set of rules used to build them, even if only approximately. Without pointing at any particular example, it is not difficult to find cases in the literature that seem to be taking this assumption for granted, either implicitly or explicitly. Specifically, one might be tempted to believe that in a Mamdani system the joint truth of the premises guarantees the truth of the conclusions. In logics that admit degrees of partial truth, this expectation would read that if the inputs of the system are true to some degree (i.e., they satisfy the antecedents of the rules to some extent), then the outputs of the system should also be true to at least the same degree (i.e., they should satisfy the consequents of the rules to at least the same extent). This fallacious interpretation of Mamdani systems as truth-preserving inference machines is certainly - and fortunately - not shared by everyone, but is reasonably widespread and does permeate many simulation applications of the technique.

1.8 To be clear, Mamdani systems are not truth-preserving in the sense stated above; they can lead to very different results from those obtained if the IF-THEN rules embedded within are interpreted as proper logical implications. This fact has already been well established in the specialized literature of fuzzy logic - as the quote below shows - but, arguably, it does not seem to be so conspicuous in many practical applications of the technique.

1.9 [The inference rule used by Mamdani systems] "is not a logical inference, i.e., a procedure aiming at the derivation of new facts from some other known ones using formal deduction rules. No logical implication is inside and thus, no modus ponens proceeds." (Klawonna \& Novák 1996.

1.10 This paper illustrates through several examples why the Mamdani method is not appropriate to explore the logical deductive consequences of a set of IF-THEN implication premises. More technical discussions of some of the aspects that we illustrate in this paper can also be found in the literature (Bodenhofer et al.2007: Dubois \& Prade 1996: Hájek 1998; Klawonna \& Novák 1996: Novák 1994.

1.11 The remaining of this paper is structured as follows. Section 2 introduces Mamdani systems and section 3 discusses their usefulness for modelling and simulation. The main objective of Section 3 is to show and illustrate with examples why Mamdani systems are not a proper tool to explore the logical consequences of IF-THEN rules; thus, they are not useful to explore the behaviour of a model defined by IF-THEN implication rules. Finally, in Section 4 we present the conclusions of the paper.

\section{Mamdani Fuzzy Systems}

2.1 Mamdani fuzzy systems were originally designed to imitate the performance of human operators in charge of controlling certain industrial processes (Mamdani 1974, 1976, 1977; Mamdani \& Assilian|1975). The aim was to summarize the operator's experience into a set of (linguistic) IF-THEN rules that could be used by a machine to automatically control the process. Specifically, using such a set of IF-THEN rules, a Mamdani fuzzy system defines a function $f$ which generates numerical outputs $y=f(x)$ from (usually numerical) input values $x$. Here 
we present a reduced and simplified exposition of the method. For a more complete and detailed presentation, the reader is referred to Sections 11.4.1 and 11.4.2 in Zimmermann 2001) or Section 11.4 and Chapter 12 in Klir \& Yuan (1995).

2.2 Mamdani systems are composed of IF-THEN rules of the form "IF $X$ is A THEN $Y$ is $B$ ", such as "IF PRESSURE is HIGH THEN VOLUME is LOW". The IF part " $X$ is $A$ " is called the antecedent of the rule, and the THEN part " $Y$ is $B$ " is called the consequent of the rule.

2.3 For simplicity in the exposition of the method and the examples, let us assume that $X$ and $Y$ (PRESSURE and VOLUME respectively in the example above) are numerical variables defined on real intervals. The examples we provide can be easily adapted to other input and output spaces, multiple inputs, or fuzzy inputs. Thus, henceforth variable $X$ is assumed to be defined in a real interval that we call the input interval, whilst variable $Y$ is assumed to be defined in a real interval that we call the output interval. Let us use lower-case letters $x$ and $y$ to denote specific values of the variables $X$ and $Y$ respectively.

2.4 The symbols $A$ and $B$ (HIGH and LOW respectively in the example above) denote linguistic terms that are modeled as fuzzy sets defined on the input and output intervals respectively. Fuzzy set $A$ is defined by a membership function $\mu_{A}$ that assigns a real value $\mu_{A}(x)$ between 0 and 1 to each element $x$ in the input interval. The value $\mu_{A}(x)$ is called the degree of membership of element $x$ in fuzzy set $A$, and can be interpreted as the extent to which element $x$ belongs to fuzzy set $A$. If the fuzzy set $A$ represents a certain concept (i.e. "HIGH"), $\mu_{A}(x)$ can also be interpreted as the truth value of the proposition " $X$ is $A$ " whenever $X=x$ (e.g. the truth value of "PRESSURE is HIGH" whenever PRESSURE $=x$ ), represented as TruthValue $(X i s A \mid X=x)$. Likewise, fuzzy set $\mathrm{B}$ is defined by a membership function $\mu_{B}$ that assigns a real value $\mu_{B}(y)$ between 0 and 1 to each real value $y$ in the output interval.

2.5 Most often Mamdani systems are composed of several IF-THEN rules. Naturally, each of the rules (which we index with subscript $k$ ) may use different fuzzy sets $A_{k}$ and $B_{k}$. The antecedents and consequents can also be combined propositions that include the logical connectives AND or OR. A standard Mamdani system uses the following operations to compute the truth value of combined propositions:

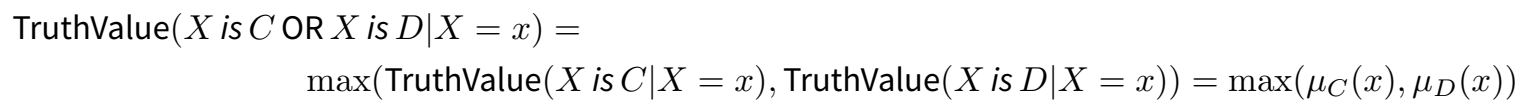

TruthValue $(X$ is $C$ AND $X$ is $D \mid X=x)=$

$$
\min (\operatorname{TruthValue}(X \text { is } C \mid X=x), \operatorname{TruthValue}(X \text { is } D \mid X=x))=\min \left(\mu_{C}(x), \mu_{D}(x)\right)
$$

The logical negation is implemented in a standard Mamdani system as follows:

$$
\text { TruthValue }(X \text { is NOT } A \mid X=x)=1-\operatorname{TruthValue}(X \text { is } A \mid X=x)=1-\mu_{A}(x)
$$

2.6 Leaving aside a possible fuzzification step, which is not relevant for our discussion, the algorithm that a Mamdani system uses to compute a numerical output $y$ from a numerical input $X=x$, given a set of rules "IF $X$ is $A_{k}$ THEN $Y$ is $B_{k}$ ", consists of the following steps:

2.7 M1. - Compute the degrees of consistency between observations (inputs) and antecedents of each rule. In this step we evaluate the extent to which the antecedent of each IF-THEN rule is satisfied for a given input. The degree of consistency between an input or observation $X=x$ and an antecedent " $X$ is $A$ " is simply the degree of membership of $x$ in the fuzzy set $A$, i.e. $\mu_{A}(x)$. The result of this step is a number $\mu_{A k}(x)$ for each rule "IF $X$ is $A_{k}$ THEN $Y$ is $B_{k}$ " (i.e. the degree of consistency between the input and each rule's antecedent). If $\mu_{A k}(x)>0$ the corresponding rule $k$ is said to be "fired".

2.8 M2. - Truncate the fuzzy set in the consequent of each rule. The result of this step for each rule "IF $X i s A_{k}$ THEN $Y$ is $B_{k}$ " is the fuzzy set $B_{k}$ truncated at the level $\mu_{A k}(x)$, i.e., a set $\mu_{\text {output } k \mid x}$ such that

$$
\mu_{\text {output } k \mid x}(y \mid x)=\min \left(\mu_{B k}(y), \mu_{A k}(x)\right)
$$

2.9 M3. - Aggregate all the truncated fuzzy sets. In this step the truncated fuzzy sets corresponding to each fired rule are aggregated to provide one single fuzzy set $\mu_{\text {Mamdani|x }}$ defined by the membership function

$$
\mu_{\text {Mamdani } \mid x}(y)=\max _{k}\left[\mu_{\text {output } k \mid x}(y)\right]=\max _{k}\left[\min \left(\mu_{B k}(y), \mu_{A k}(x)\right)\right]
$$

2.10 The equation above clearly shows why Mamdani fuzzy systems are sometimes called max-min fuzzy systems. 
2.11 M4. - Defuzzify the aggregated fuzzy set. The defuzzification step transforms the aggregated fuzzy set $\mu_{\text {Mamdani } \mid x}$ into one single crisp number. Standard Mamdani systems use the Centre of Gravity (COG) defuzzification method. This method returns the projection (on the horizontal axis) of the centre of gravity of the area under the membership function $\mu_{\text {Mamdani|x }}$. If some input value is such that no rule is fired, the centre of gravity for $\mu_{\text {Mamdani|x }}$ cannot be calculated. In that case, some default output value can be considered, or the system can be readjusted to avoid that situation (e.g. by modifying the fuzzy sets $A_{k}$, or by including new rules).

2.12 Given that the defuzzification step has a large influence on the final function that the system provides, we will also consider here two other alternative defuzzification methods (Van Leekwijck \& Kerre 1999): i) First of Maxima. This method returns the smallest value of $y$ for which the membership function $\mu_{\text {Mamdani } \mid x}$ attains its maximum value. ii) Last of Maxima. This method returns the greatest value of $y$ for which the membership function $\mu_{\text {Mamdani|x }}$ attains its maximum value.

\section{Modelling Using Mamdani Fuzzy Systems}

3.1 We consider two different potential objectives when modelling with Mamdani fuzzy systems: a) Modelling with the purpose of defining, capturing or reproducing some input-output function. b) Modelling with the purpose of exploring the logical consequences of an IF-THEN rule or of a set of IF-THEN rules.

3.2 As to the first objective, Mamdani systems have proven to be a very useful tool for defining or approximating functions based on IF-THEN rules, and they have been quite successful on practical applications, mainly in the field of control systems (Driankov et al. 1996, Thirumoorthi \& Yadaiah 2015; Zimmermann 2001). Some of the appealing features of Mamdani systems for this purpose are: i) Flexibility: Mamdani-type fuzzy controllers are universal fuzzy controllers (Cao et al. 2001). ii) They provide a natural framework to include expert knowledge in the form of linguistic rules (Cordón et al.|2001). iii) Local adaptability: it is possible to define rules that are fired only at some specific regions of the input space. This allows for local adaptations of the working function on those particular regions without modifying the function outside those regions (Mamdanil1994).

3.3 Henceforth we will focus our discussion on the second potential objective of modelling with IF-THEN rules, namely exploring the behaviour or consequences of a model which is characterized by a set of IF-THEN rules. Our objective is to illustrate and explain why Mamdani systems are dangerous tools for that purpose. To that end, we will first discuss the effect of IF-THEN rules in Mamdani systems. We are interested in the total inputoutput effect. Thus, even though we will sometimes refer to the intermediate steps where the fuzzy consequents of each IF-THEN rule are truncated, we will always be considering the final input-output effect of a rule after the system has gone through all its steps, including aggregation and defuzzification. This tactic complements the approach usually followed in fuzzy logic, which has typically focused on the study of each individual step of the process in isolation. By contrast, here we study the properties of the final function provided by the Mamdani system as a whole, treating its inner intermediate steps as black boxes.

3.4 Thus, the question is whether an IF-THEN rule of the form "IF $X$ is A THEN $Y$ is B" in a Mamdani system imposes the same kind of input-output relation that one could expect if the rule were to be interpreted as a logical implication. Specifically, given the rule "IF $X$ is $A$ THEN $Y$ is $B$ ", and interpreting $\mu_{A}(x)$ as the truth value of proposition " $X$ is $A$ " whenever $X=x$, we aim to find out whether providing any input $x$ with high degree of membership in $A$ (high $\mu_{A}(x)$ ) guarantees that its corresponding output $y(x)$ will have a high degree of membership in $B$ (high $\mu_{B}(y(x))$ ), which we consider to be the relation that one would expect if the rule were interpreted as a logical implication.

3.5 More precisely, consider a Mamdani system with the rule "IF $X$ is $A$ THEN $Y$ is $B$ ". This system defines a function $y$ that assigns a unique output value $y(x)$ to each input value $x$. We aim to determine whether the presence of the rule "IF $X$ is $A$ THEN $Y$ is $B$ " in the system guarantees that the constraint $\mu_{B}(y(x)) \geq \mu_{A}(x)$ is satisfied for all inputs $x$, either strictly or approximately in some sense 1 . Considering that the implication connective $a \rightarrow c$ tries to quantify the degree by which the consequent $c$ is at least as true as the antecedent $a$ Hájek 1998 Smets \& Magrez 1987), we will refer to the previous condition as the logical-implication interpretation of the rule "IF $X$ is $A$ THEN $Y$ is $B$ ".

3.6 We will start by discussing systems with a single rule, and then we will discuss systems with more rules. It must be said beforehand that Mamdani systems with only one rule are generally not used. The reason is that each individual rule in a Mamdani system is intended to shape the final function somewhat locally, i.e. individual rules modify the input-output relation on specific regions of the input space without necessarily altering the relation on other regions (i.e. those where the rule does not apply) (Bodenhofer et al.|2007). Thus, a system with a single rule will usually leave the input-output relationship undefined where the rule is not fired, and, even if 
fired, one single rule will usually fail to capture any minimally complex association. Nonetheless, discussing systems with a single rule will be instructive because they are simple and, at the same time, sufficient to show the difference between the Mamdani effects and the logical-implication effects of an IF-THEN rule. Furthermore, since single-rule systems lack the aggregation step, their analysis provides a neater understanding of the other steps of the Mamdani process (most notably the defuzzification step, which is pivotal in our arguments).

\section{Single-rule systems}

3.7 In this section we study a standard Mamdani system with one single rule "IF $X$ is $A$ THEN $Y$ is $B$ ". This system defines a function $y$ which, given an input $x$, produces an output $y(x)$. It is shown below that the function $y$ provided by the Mamdani system does not necessarily satisfy the constraint $\mu_{B}(y(x)) \geq \mu_{A}(x)$ for all inputs $x$ (i.e. the logical-implication interpretation of the rule), neither precisely nor approximately, even in those cases in which there does exist one or multiple functions that do satisfy the constraint.

3.8 In order to work with a concrete example let us suppose that $X$ and $Y$ take real values in the interval [0, 10], and let us consider the rule "IF $X$ is large THEN $Y$ is large", where the fuzzy set "large" is defined in the interval $[0,10]$ by the membership function $\mu_{\text {large }}(z)=0.1 z$ for both variables $X$ and $Y$ (Figure 1 ).

3.9 We discuss the following question: Is a Mamdani system a proper tool to explore the logical consequences of an IF-THEN rule about the variables in the system? To this end, first we consider the difference between the logical consequences of the rule and one of the logically consistent outputs.

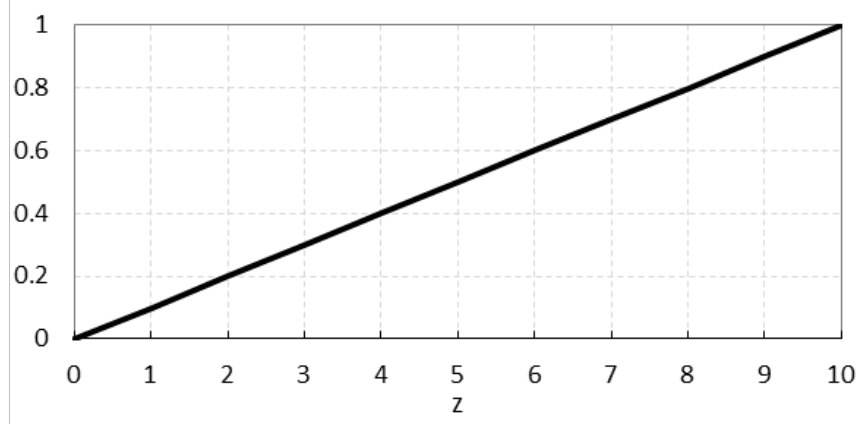

Figure 1: Membership function of the fuzzy set 'large"

3.10 Suppose that we assume that a Mamdani system will provide the input-output relationship implied by the rule "IF $X$ is large THEN $Y$ is large". Note that there is already some a priori impossibility result in the response to this question, because in general a logical IF-THEN rule does not define any specific function (which is what a Mamdani system will return), but it only rules out some input-output value combinations (in crisp logic, those combinations which make the antecedent TRUE and the consequent FALSE). Any specific function will necessarily leave out any other alternative functions that may be equally compatible with the logical rule. If we consider that the functions compatible with the rule "IF $X$ is large THEN $Y$ is large" are those functions $f$ such that $\mu_{\text {large }}(f(x)) \geq \mu_{\text {large }}(x)$ for all inputs $x$, then it is clear that there are many possible functions compatible with the rule. In fact, any function such that $f(x) \geq x$ for all inputs $x$ in the range $[0,10]$ will do (for instance, the constant function defined by $f(x)=10$, or any of the functions represented in Figure 2. Any function "drawn" above the dashed diagonal lines in Figure 2 would be compatible with this rule. 

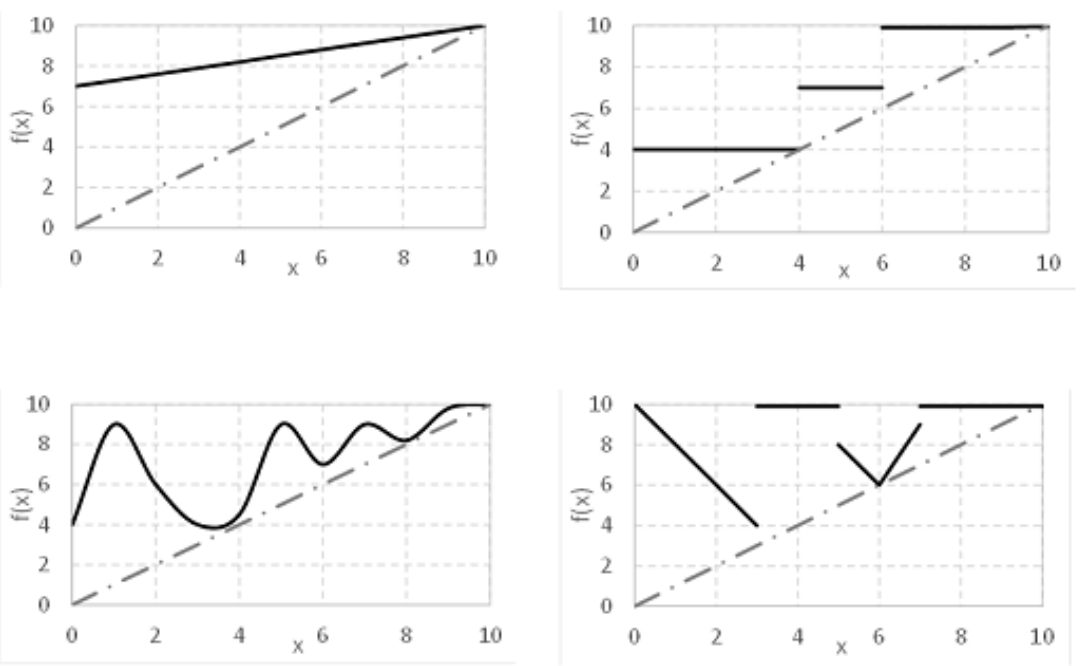

Figure 2: Rule-compatible functions. Different functions $f$ fully compatible with the rule "IF $X$ is large THEN $Y$ is large" in the sense that they satisfy the constraint $\mu_{\text {large }}(f(x)) \geq \mu_{\text {large }}(x)$.

3.11 Note the risks of considering any of the rule-compatible functions represented in Figure 2 as if it were "the" logical consequent function of the IF-THEN rule, or as if it were characterizing the logical implication of the IFTHEN rule. Any system that provides a function as the result of an IF-THEN rule should be suspicious in the sense that it is probably making many additional assumptions about the input-output relationship than just those corresponding to the logical-implication interpretation of the IF-THEN rule.

3.12 To illustrate this point further, consider for instance the crisp rule "IF $X \leq 2$ THEN $Y \geq 8$ ". Note that looking for "the logical consequent function" derived from this rule is something that does not make much sense. What output value should such a theoretical function provide for an input value $x=1$ ? The constraint "IF $X \leq 2$ THEN $Y \geq 8$ " indicates that certain output values are not allowed, but it does not specify what "the" output value should be.

3.13 In general, choosing one particular value that is consistent with a rule is a very different exercise from that of exploring the logical consequences of the rule. Suppose, for instance, that the evidence of a murder scene indicates that the killer was a dog. An inspector who takes the first dog he sees and delivers it as the murderer is providing an output (i.e. the dog as the killer) that is consistent with the evidence, but taking that particular dog as the murderer can hardly be called "the logical consequence" of the evidence.

3.14 Going back to our example with the rule "IF $X$ is large THEN $Y$ is large", we already know that there are infinite functions $f$ that satisfy the constraint $\mu_{\text {large }}(f(x)) \geq \mu_{\text {large }}(x)$ for all inputs $x$. However, a standard Mamdani system (with COG defuzzification) with the rule "IF $X$ is large THEN $Y$ is large" provides the function $y$ represented in Figure 3, which, for a large range of input values, is not compatible with the logical-implication interpretation of the rule, i.e., it is not even one of the infinite possible functions that do satisfy the constraint $\mu_{\text {large }}(y(x)) \geq \mu_{\text {large }}(x)$. 


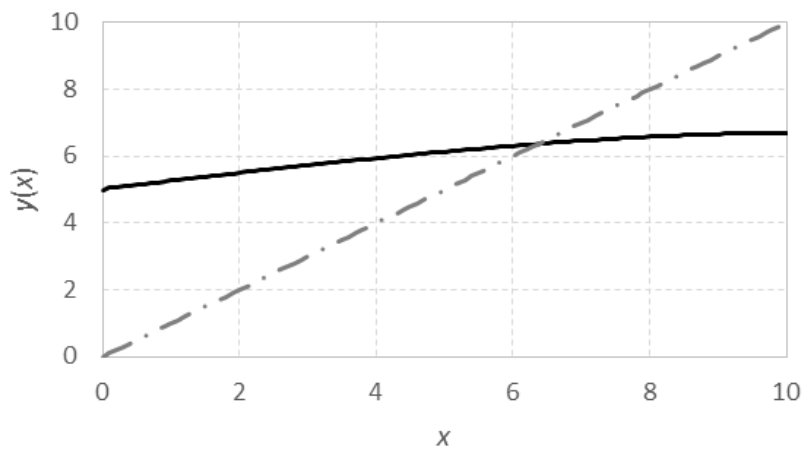

Figure 3: Mamdani function. Representation of the function generated by a Mamdani system with the rule "IF $X$ is large THEN $Y$ is large". The points below the dashed diagonal do not satisfy the logical implication constraint $\mu_{\text {large }}(y(x)) \geq \mu_{\text {large }}(x)$.

3.15 It must be noted here that, in the single rule case, a Mamdani-like system with one of the so-called maxima defuzzification methods (Van Leekwijck \& Kerre 1999) such as First of Maxima or Last of Maxima, instead of Centre of Gravity, would provide one of the (possibly many, if they exist ${ }^{2}$ ) functions $y$ satisfying the constraint $\mu_{B}(y(x)) \geq \mu_{A}(x)$ for all inputs $x^{3}$. However, each defuzzification method has advantages and drawbacks Hellendoorn \& Thomas 1993; Klir \& Yuan 1995; Van Leekwijck \& Kerre 1999: Ross 2010, Zimmermann 2001). The maxima methods are usually considered to be better candidates for fuzzy reasoning systems, while the Centre of Gravity method and other area methods exhibit the property of continuity, which makes them more suitable for fuzzy controllers (Van Leekwijck \& Kerre 1999).

3.16 In short, for a standard Mamdani system we arrive at the following result:

3.17 Caveat 1: The function $y$ produced by a standard Mamdani system with rule "IF $X$ is $A$ THEN $Y$ is $B$ " does not necessarily satisfy the constraint $\mu_{B}(y(x)) \geq \mu_{A}(x)$ for all inputs $x$, even if there exist functions that do satisfy the constraint.

3.18 The following example will illustrate that a standard Mamdani system with rule "IF $X$ is $A$ then $Y$ is $B$ " may not comply with the logical-implication interpretation of its rule at all, i.e. not even in the approximate sense that any input $x$ with high membership in $A$ - high $\mu_{A}(x)$ - should guarantee an output $y(x)$ with high membership in $B$ - high $\mu_{B}(y(x))$.

3.19 Let us consider the rule "IF $X$ is large THEN $Y$ is NOT about five", where the fuzzy set 'about five" is defined in the interval $[0,10]$ as shown in Figure 4 and the fuzzy set "NOT about five" is defined by the membership function $\mu_{\mathrm{NOT} \text { about five }}(y)=1-\mu_{\text {about five }}(y)$.
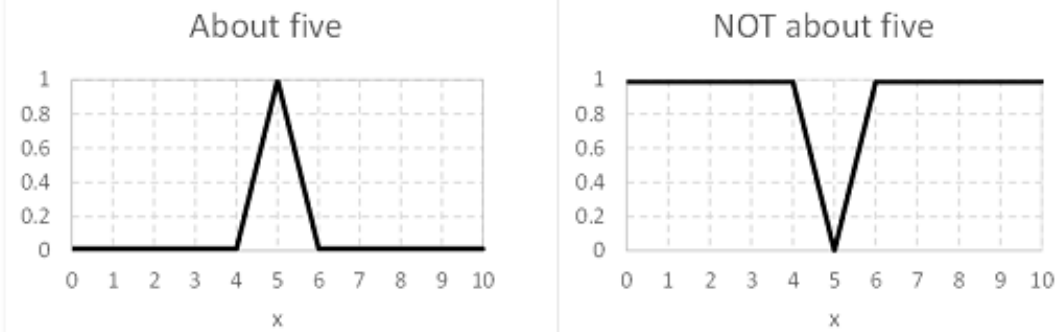

Figure 4: Membership functions for the fuzzy sets "About five" (left) and "NOT about five" (right).

3.20 Considering that the consequent fuzzy set "NOT about five" is defined by a membership function that is symmetric around $y=5$, the output of a Mamdani system with the rule "IF $X$ is large THEN $Y$ is NOT about five" for any input value $x$ that fires the rule is precisely $y(x)=5$, i.e., the very value that does not satisfy the consequent of the rule to any degree.

3.21 The purpose of the previous example is to show that, in a Mamdani system with the rule "IF $X$ is $A$ THEN $Y$ is $B$ ", an input $x$ may satisfy the antecedent to a large or even total degree $\left(\mu_{A}(x)=1\right)$ but its corresponding 
output $y(x)$ may not satisfy the consequent to any degree whatsoever $\left(\mu_{B}(y(x))=0\right)$. We can draw two important lessons from this observation. The first lesson is that there are Mamdani systems for which the constraint $\mu_{B}(y(x)) \geq \mu_{A}(x)$ (i.e. the logical-implication interpretation of the rule) is as far from being valid as it can possibly be, at least for some inputs. Thus, we can state that, in general, Mamdani systems do not necessarily satisfy the logical-implication interpretation of their rules, neither precisely nor approximately. Specifically, we can issue the following two caveats, which could be considered the fuzzy extensions of Caveat 1.

3.22 Caveat 2. In a standard Mamdani system with rule "IF $X$ is $A$ THEN $Y$ is $B$ ", an input $\mathrm{x}$ with high degree of membership in $A$ (high $\mu_{A}(x)$ ) does not guarantee an output $y(x)$ with high degree of membership in $B$ (high $\left.\mu_{B}(y(x))\right)$.

3.23 Caveat 3. In a standard Mamdani system with rule "IF $X$ is $A$ THEN $Y$ is $B$ ", the observation of an output $y(x)$ with low degree of membership in $B$ (low $\mu_{B}(y(x))$ ) does not guarantee that its corresponding input(s) $x$ have low degree of membership in $A$ (low $\mu_{A}(x)$ ).

3.24 The second lesson we can draw from the example is that neither the rule of inference modus ponens (which allows inferring that the consequent will be true whenever the antecedent is true) nor the rule of inference modus tollens (which allows inferring that the antecedent is false whenever we observe that the consequent is false) can be safely applied when dealing with Mamdani systems. In other words, in Mamdani systems with one rule of the form "If Antecedent THEN Consequent":

a) If the Antecedent is fully true, it cannot be inferred that the Consequent must be true; the Consequent could be completely false (i.e. modus ponens does not apply).

b) If the Consequent is fully false, it cannot be inferred that the Antecedent must be false; the Antecedent could be completely true (i.e. modus tollens does not apply).

3.25 Basically, Mamdani systems include information about the function that one wants to obtain using the same language of logical implications, but the real effect of an "IF $X$ is $A$ THEN $Y$ is $B$ " rule over the obtained function can be very different from its expected effect if it is understood as a logical implication.

3.26 In summary, a Mamdani system with a single rule "IF $X$ is $A$ THEN $Y$ is $B$ " provides a function $y$ in the support of the antecedent which, depending on the defuzzification method, will be one of the (possibly infinite) functions $f$ that satisfies the logical implication constraint (i.e. $\mu_{B}(f(x)) \geq \mu_{A}(x)$ for all inputs $x$ ), or a function that does not even meet that condition, neither exactly nor approximately, even if there are available functions that do satisfy the condition. In either case, it seems clear that these systems do not constitute a proper tool to explore the logical consequences of an IF-THEN implication premise.

3.27 Naturally, this general result does not preclude the fact that, in certain particular cases or under additional constraints, the outputs provided by a Mamdani system may "make sense" when the rules are interpreted as logical implications. Some scholars have studied conditions under which these systems can be more amenable to admit some "interpretability", although the word "interpretability" is often used in an intuitive and informal sense (Alonso \& Magdalena 2011; Alonso et al. 2009, Cordón 2011, Jin 2003; Moraga 2012). The definition and measure of interpretability is an active current trend of research in the field (Alonso et al. 2015).

3.28 In the following section we discuss the multi-rule case, where - regardless of the defuzzification method - the logical implication constraint (i.e. $\mu_{B k}(y(x)) \geq \mu_{A k}(x)$ for all inputs $x$ ) is not guaranteed for every rule in the system, not even in those cases in which there exist functions that do satisfy all such constraints. This is due to the way aggregation is carried out in a Mamdani system.

\section{Systems with multiple rules}

3.29 Consider a Mamdani system with the following two rules:

IF $X$ is approximately less than three THEN $Y$ is greater than five

IF $X$ is approximately less than two THEN $Y$ is about nine

3.30 Where the fuzzy sets "approximately less than three", "greater than five", "approximately less than two" and "about nine" are represented in Figure 5 . What output could we expect from this system for low input values, e.g. for $x$ less than one?

3.31 It seems clear that any input $x \leq 1$ satisfies completely the antecedents of both rules, so if we interpret the rules as two logical implications, and we want the output to satisfy both rules completely, the corresponding output value should be $y=9$. In contrast, a Mamdani system provides an output value $y=7.47$. 

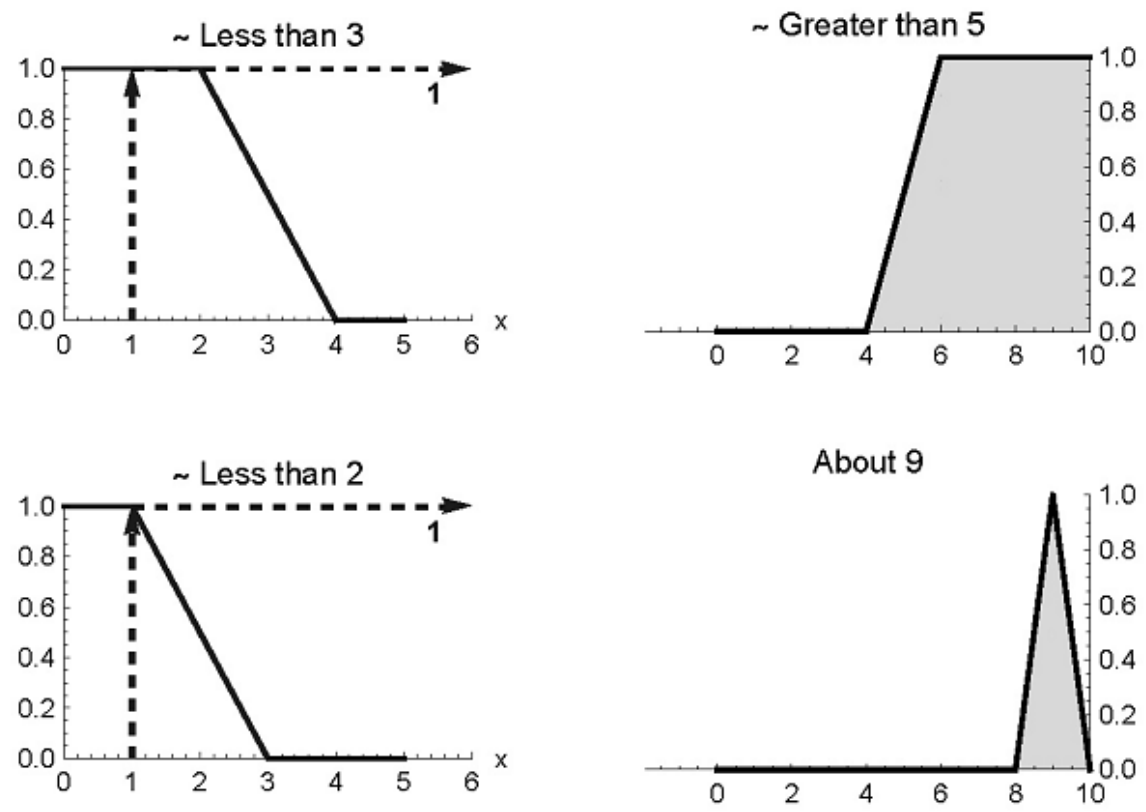

INPUT: $x=1$

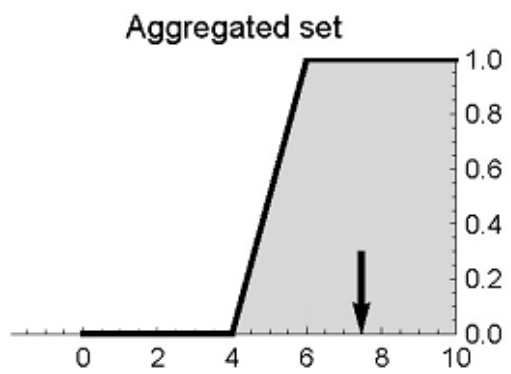

Figure 5: Mamdani process. The input value, the truncated output fuzzy sets for each rule and the final output of the system are represented.

3.32 Let us consider the aggregation step, represented in Figure 5 . Given that for $x \leq 1$ the antecedents of both rules are satisfied completely, the result of the first rule is precisely the fuzzy set "greater than five" and the result of the second rule is the fuzzy set "about nine" (see Figure 5. The use of the function MAX as an aggregation method in Mamdani systems provides in this case an output that coincides with the fuzzy set "greater than five" and loses the information in "about nine".

3.33 Naturally, the reason is that the aggregation step in a Mamdani system is not intended to produce outputs that satisfy all the IF-THEN implication rules. In some cases (e.g. if a maxima defuzzification method such as First of Maxima or Last of Maxima is used), the Mamdani system will provide an output in accordance with at least one of the IF-THEN rules that the input fires (assuming that at least one rule is fired), i.e., an output value $y(x)$ such that $\mu_{B k}(y(x)) \geq \mu_{A k}(x)$ for some rule "IF $X$ is $A_{k}$ THEN $Y$ is $B_{k}$ "; however, that very output $y(x)$ could be simultaneously violating the constraints imposed by the other rules. That is indeed the case in our example: for an input $x=1$, the First of Maxima defuzzification method provides $y(1)=6$, and the Last of Maxima defuzzification method provides $y(1)=10$. Both values satisfy $\mu_{B}(y(1))=1 \geq \mu_{A}(1)=1$ for the rule "IF $X$ is approximately less than three THEN $Y$ is greater than five", but none of them satisfies the corresponding relation for the rule "IF $X$ is approximately less than two THEN $Y$ is about nine". In fact, with both methods the input satisfies the antecedent of this latter rule completely, but the output does not satisfy the corresponding consequent of the rule to any degree whatsoever $\left(\mu_{\text {About9 }}(y(1))=0<\mu_{\sim \text { LessThan2 }}(1)=1\right)$.

3.34 If the COG defuzzification method is used, the selected output can be interpreted as a compromise or interpolated value between output regions (fuzzy sets), but that interpolated value may not itself belong to any of the regions, i.e., it may not satisfy any of the logical-implication constraints to any degree at all.

3.35 In short, it is clear then that the MAX aggregation method of Mamdani systems is not consistent with a conjunc- 
tive interpretation of the set of IF-THEN rules. In fact, a rule such as

\section{IF $X$ is outlier THEN $Y$ is very small OR $Y$ is very large}

is equivalent in a Mamdani system (using the MAX function for logical OR) to the set of rules:

$$
\begin{aligned}
& \text { IF } X \text { is outlier THEN } Y \text { is very small } \\
& \text { IF } X \text { is outlier THEN } Y \text { is very large }
\end{aligned}
$$

3.36 So the effect of a set of rules in a Mamdani system is disjunctive rather than conjunctive (Dubois \& Prade 1996). Incidentally, in this example, for an input x with a high degree of membership in the fuzzy set "outlier", the output of a Mamdani system with the COG defuzzification method would be an interpolation or compromise between the "very small" and "very large" regions, and the result of this compromise will likely not belong to any of the extreme regions, i.e., it may not satisfy the consequent of any rule to any degree.

3.37 Thus, we summarize this point in the following caveat.

Caveat 4. In a standard Mamdani system with several rules of the form "IF $X$ is $A_{k}$ THEN $Y$ is $B_{k}$ ", an input $x$ that complies with the antecedents of all the rules to a great extent (high $\mu_{A k}(x)$ for all $k$ ) may lead to an output $y$ which does not satisfy the consequent of any of the rules to any extent (low $\mu_{B k}(y(x))$ for all $k$ ).

3.38 A crucial consequence of the MAX aggregation method is that IF-THEN rules in a Mamdani system have effects on the final output of the system that cannot be analyzed independently of other IF-THEN rules that may be fired simultaneously in the system.

3.39 Finally, it should be noted here that, as indicated for single-rule systems, one could consider additional constraints on the definitions of the fuzzy sets and on the rule base to try to avoid the type of effects that we have discussed here, and thus attempt to make the system more amenable to some notion of interpretability Alonso et al. 2009, Cordón 2011; Jin 2003. Nonetheless, in the absence of such efforts, it is clear that regarding Mamdani systems as truth-preserving inference machines - even if only approximately - is certainly not appropriate.

\section{Conclusions}

4.1 This paper discusses the usefulness of Mamdani fuzzy systems for modelling, simulation and decision making. We have considered two different objectives of the modelling process. The first objective is the approximation or definition of a function using information in the form of linguistic IF-THEN rules. The second objective is the exploration of the logical implications of a model defined by a set of IF-THEN rules that express some causality or relation between two (or more) variables $X$ and $Y$.

4.2 As to the first objective, it is clear that Mamdani systems have proven successful for function approximation in many practical applications (Azzini et al. 2008, Behnamian 2015, Elragal 2014, Shankar et al. 2015, Yang et al. 2015), especially in fuzzy control. Their success is mainly derived from two characteristics that are very convenient for this objective: Mamdani systems are universal approximators and can include knowledge in the form of linguistic rules that can be used for local fine-tuning.

4.3 By contrast, the use of Mamdani systems for the second objective stated above is questionable Klawonna \& Novák 1996. The bottom line is that a Mamdani system with a rule "IF $X$ is $A$ THEN $Y$ is $B$ " can generally fail to assign outputs with high membership in $B$ to inputs with high membership in $A$. This failure is a consequence of two operations that take place within Mamdani systems: defuzzification and aggregation.

4.4 The COG defuzzification method used in standard Mamdani systems does not guarantee that the output will satisfy the consequent of an IF-THEN rule to at least the same degree that the input satisfies the antecedent. In fact, in the general case, it does not guarantee that the output will satisfy the consequent to any degree at all, regardless of how much the input satisfies the antecedent.

4.5 As for the aggregation of rules, the MAX operator used in standard Mamdani systems does not permit an interpretation of the IF-THEN rules as independent implication constraints which must be simultaneously (i.e. conjunctively) satisfied - either completely or, in some sense, to the highest possible common degree. This method aggregates the output sets of each fired rule as if they were disjunctive rules; if the rules are understood as constraints on the output values, this means that some of the constraints, even if fired, may not be effective at all. As a consequence, an individual rule, however sharp or restrictive it may be when interpreted as an implication constraint, may not actually impose any actual constraint or have any effect whatsoever on 
the output of the Mamdani system (see Sections 3.28-3.34). This can certainly generate some confusion, either when trying to interpret the set of rules of a Mamdani system or when trying to incorporate expert or previous knowledge in the system: "The almost systematic use of conjunction-based representations, since Mamdani's early works, including Sugeno's fuzzy rules with precise conclusions, is surprising from the point of view of logic: conjunction-based rules do not fit with the usual meaning of rules in expert systems" (Dubois \& Prade 1996).

4.6 It must be noted here that other fuzzy-logic-based approaches to function approximation with IF-THEN rules are possible (Hájek 1998, 2010, Jones et al.|2009, Klawonna \& Novák 1996), and that some of these approaches would obtain a) one of the functions that do satisfy all the IF-THEN rule implication constraints (in the sense $\mu_{B k}(y(x)) \geq \mu_{A k}(x)$ for all rules $k$ ) whenever such functions exist, or b) one of the functions that best satisfy the implication constraints according to some criteria, whenever complete satisfaction is not possible. This approach, based on fuzzy rules interpreted as logical implications, has been termed deductive interpretation Bodenhofer et al.2007) or implication-based model (Dubois \& Prade 1996).

4.7 In more general terms, one should always be wary of interpreting methods that produce functions (i.e., correspondences that assign one unique output value to each input value) from IF-THEN rules, as if they were providing the logical consequences of the rules. If by "the logical consequences of the rules" we understand the set of values $y(x)$ that for a given input $x$, are consistent with the IF-THEN rules as logical implications (i.e. those values $y(x)$ that satisfy $\mu_{B k}(y(x)) \geq \mu_{A k}(x)$ for all rules $k$ either strictly or approximately in some sense) then, in most cases, the set of consistent values will not define a unique function, and any system that in such cases provides a particular input-output function will possibly be making - either implicitly or explicitly strong assumptions about the behaviour of the system beyond those embedded in the IF-THEN rules and the membership functions. This important aspect of the function-approximation techniques is often ignored in the literature on fuzzy control and approximate reasoning: "Various books explaining fuzzy control, written by nonlogicians, suffer by logical mismatch caused by the fact that "fuzzy IF-THEN rules" are presented as implications but then used to construct a fuzzy relation having little to do with any implication, at least at first glance" Hájek 1998.

4.8 As Novák (2012) indicates about the function-approximation approach, "This method of interpretation of fuzzy IF-THEN rules is very convenient when we need a nice tool for the approximation of functions but it is less convenient as a model of human reasoning". Such models can also be very sensitive to apparently irrelevant choices in the fuzzy inference process, particularly those pertaining to the defuzzification method.

4.9 To conclude, suppose that we use a Mamdani-like system to simulate and explore the behaviour of a model defined by a set of IF-THEN rules. If by consistency of an outcome we understand that the outcome satisfies the consequents of the rules to at least the same degree as its corresponding input satisfies the antecedents, then any simulation based on IF-THEN rules and on this type of methods that obtain functions from rules will be providing, in the best-case scenario - for instance, if deductive inference (Bodenhofer et al. 2007) is used -, just one of the (possibly many and possibly very different) outcomes that are consistent - or in some sense most consistent - with the IF-THEN rules; in the worst-case scenario, which may happen for instance if a Mamdani approach is used, it will be providing an outcome that may not belong to the set of most-consistent outcomes and which in fact may even be highly inconsistent with the rules.

4.10 Obtaining a unique output from observations and rules can be a potentially desired objective of a logical process, but the selection of that unique output should not be based on arbitrary steps. Sherlock Holmes famously stated: "It is an old maxim of mine that when you have excluded the impossible, whatever remains, however improbable, must be the truth." (Doyle 1892). Note that the key to finding the explanation or the consequence of the evidence is not choosing one particular explanation that is consistent - or, in some sense, the most consistent - with the currently available facts, but eliminating alternative explanations that have not been eliminated yet, a process which may require the acquisition of new evidence. Whenever the available evidence points to a broad set of possible culprits of a crime, it is difficult to think that Holmes would be happy by just choosing any of them as the culprit. If a witness indicates that the criminal "was tall", the statement "his height is 2 meters" might be considered one of the most consistent statements with relation to the evidence, but it seems very unsatisfactory and misleading to say that it would be the logical consequence of the testimony. In a sense, defuzzification, or choosing one particular value from a set of possible values plays this role of selecting one particular culprit when the evidence equally points to many ones, or when it has little discriminatory power among them. Defuzzification is needed if one is looking for a unique output value corresponding to an input value but, as Leekwijck and Kerre indicate Van Leekwijck \& Kerre 1999, "the whole concept of defuzzication is completely opposite to the main purpose of fuzzy set theory namely the extension of crisp concepts and theories".

4.11 We conclude this assessment with a few practical take-away messages. Suppose that you are considering a Mamdani system to capture the decision procedure of some agent in your model: 
1. If the decision procedure is originally characterised by logical IF-THEN rules, i.e., if those rules are the axioms or primitives that define the decision procedure of the agent, then, Mamdani systems are not a safe tool to explore the expected logical consequences of those rules: without further validation, they may not provide what one could expect according to almost any sensible interpretation of those rules.

2. However, if the decision function of an agent, understood as an inputs-output relationship, is known (or if there is enough data), Mamdani systems can be safely used to approximate and "store" that function. Note that this is a function-approximation approach (based on adjusting data points), in contrast with the deductive approach (based on obtaining the logical consequences of the rules).

3. If some degree of interpretability or logical link between IF-THEN rules and outputs in a Mamdani system is desired, one should consider additional constraints to those required by a standard Mamdani system, or alternative fuzzy-logic techniques that try to preserve interpretability to a greater degree (Bodenhofer et al.2007).

\section{Acknowledgements}

The authors are very grateful to Doina Olaru for many productive discussions and several comments. This research was partly supported under Australian Research Council's Discovery Projects funding scheme (project number DP130100570, “Modelling Network Innovation Performance Capability: A Multidisciplinary Approach”), Spanish Ministry of Science and Innovation's project CSD2010-00034 (SIMULPAST), project ECO2017-83147-C22-P (MINECO/AEI/FEDER, UE) and project ECO2017-83147-C2-1-P (MINECO/ AEI/FEDER, UE).

\section{Notes}

${ }^{1}$ If for a given input $x$ there is no possible output value $y(x)$ such that $\mu_{B}(y(x)) \geq \mu_{A}(x)$, we can check whether the obtained output is one of the values in the output range that minimize $\mu_{A}(x)-\mu_{B}(y(x))$.

${ }^{2}$ If the fuzzy set $B$ in the consequent of the IF-THEN rule is normal, i.e., if there is some value $y^{*}$ (possibly not unique) such that $\mu_{B}\left(y^{*}\right)=1$, then it is clear that there exist functions, such as the constant functions defined by $y(x)=y^{*}$, which do satisfy the constraint $\mu_{B}(y(x)) \geq \mu_{A}(x)$ for all inputs $\mathrm{x}$.

${ }^{3}$ One could also consider additional constraints in the desired functions, such as continuity, or the property that the greater the degree of satisfaction of the antecedent, the greater the degree of satisfaction of the consequent: $\mu_{A}\left(x_{1}\right)>\mu_{A}\left(x_{0}\right) \rightarrow \mu_{B}\left(y\left(x_{1}\right)\right)>\mu_{B}\left(y\left(x_{0}\right)\right)$ for all inputs $x_{0}, x_{1}$.

\section{References}

Alonso, J. M., Castiello, C. \& Mencar, C. (2015). Interpretability of fuzzy systems: Current research trends and prospects. In J. Kacprzyk \& W. Pedrycz (Eds.), Springer Handbook of Computational Intelligence, (pp. 219237). Berlin/Heidelberg: Springer

Alonso, J. M. \& Magdalena, L. (2011). Special issue on interpretable fuzzy systems. Information Sciences, 181(20), 4331-4339

Alonso, J. M., Magdalena, L. \& González-Rodríguez, G. (2009). Looking for a good fuzzy system interpretability index: An experimental approach. International Journal of Approximate Reasoning, 51(1), 115-134

Anand, S., Sharma, I. \& Goyal, R. (2017). Extended understanding of dyadic friendship using fuzzy measures: A simulation approach. IEEE Access, 5, 21179-21192

Arfi, B. (2010). Linguistic Fuzzy Logic Methods in Social Sciences. Berlin/Heidelberg: Springer

Azzini, A., Marrara, S., Sassi, R. \& Scotti, F. (2008). A fuzzy approach to multimodal biometric continuous authentication. Fuzzy Optimization and Decision Making, 7(3), 243

Behnamian, J. (2015). Survey on fuzzy shop scheduling. Fuzzy Optimization and Decision Making, 15(3), $331-366$ 
Bodenhofer, U., Danková, M., Stepnicka, M. \& Novák, V. (2007). A plea for the usefulness of the deductive interpretation of fuzzy rules in engineering applications. In Fuzzy Systems Conference, 2007, (pp. 1-6). IEEE International

Bodenhofer, U., Danková, M., Stepnicka, M. \& Novák, V. (2012). Fuzzy logic in financial management. In E. Dadios (Ed.), Fuzzy Logic - Emerging Technologies and Applications, (pp. 259-286). InTech

Bojadziev, G. (2007). Fuzzy Logic for Business, Finance, and Management. River Edge, NJ: World Scientific

Cao, S.-G., Rees, N. W. \& Feng, G. (2001). Mamdani-type fuzzy controllers are universal fuzzy controllers. Fuzzy Sets and Systems, 123(3), 359-367

Cioffi-Revilla, C. A. (1981). Fuzzy sets and models of international relations. American Journal of Political Science, 25(1), 129-159

Cordón, O. (2011). A historical review of evolutionary learning methods for mamdani-type fuzzy rule-based systems: Designing interpretable genetic fuzzy systems. International Journal of Approximate Reasoning, 52(6), 894-913

Cordón, O., Herrera, F., Hoffmann, F. \& Magdalena, L. (2001). Evolutionary tuning and learning of fuzzy knowledge bases. Genetic Fuzzy Systems, 19

Darbra, R. M., Eljarrat, E. \& Barceló, D. (2008). How to measure uncertainties in environmental risk assessment. TrAC Trends in Analytical Chemistry, 27(4), 377-385

Denize, S., Purchase, S. \& Olaru, D. (2012). Using case data to ensure 'real world' input validation within fuzzy set theory models. In Fuzzy Methods for Customer Relationship Management and Marketing: Applications and Classification, (pp. 61-89). IGI Global

Doyle, A. C. (1892). The adventures of sherlock holmes: The adventure of the beryl coronet. The Strand Magazine, 17

Driankov, D., Hellendoorn, H. \& Reinfrank, M. (1996). An Introduction to Fuzzy Control (2nd ed.). Berlin/Heidelberg: Springer

Dubois, D. \& Prade, H. (1996). What are fuzzy rules and how to use them. Fuzzy Sets and Systems, 84(2), 169-185

Dykstra, P., Jager, W., Elsenbroich, C., Verbrugge, R. \& de Lavalette, G. R. (2015). An agent-based dialogical model with fuzzy attitudes. Journal of Artificial Societies and Social Simulation, 18(3), 3

Elragal, H. M. (2014). Mamdani and Takagi-Sugeno fuzzy classifier accuracy improvement using enhanced particle swarm optimization. Journal of Intelligent \& Fuzzy Systems, 26(5), 2445-2457

Fleischmann, A. (2005). A model for a simple Luhmann economy. Journal of Artificial Societies and Social Simulation, $8(2), 4$

Fort, H. \& Pèrez, N. (2005). The fate of spatial dilemmas with different fuzzy measures of success. Journal of Artificial Societies and Social Simulation, 8(3), 1

Góngora y Moreno, S. F. \& Gutierrez-Garcia, J. O. (2017). Collective action in organizational structures. Computational and Mathematical Organization Theory, 24(1), 1-33

Hájek, P. (1998). Metamathematics of Fuzzy Logic (Vol. 4). Dordrecht: Springer

Hájek, P. (2010). Fuzzy logic. In The Stanford Encyclopedia of Philosophy (Fall 2010 Edition). Edward N. Zalta (ed.).

Hassan, S., Garmendia, L. \& Pavón, J. (2007). Agent-based social modeling and simulation with fuzzy sets. In E. Corchado, J. M. Corchado \& A. Abraham (Eds.), Advances in Soft Computing (Vol. 44), (pp. 40-47). Berlin/Heidelberg: Springer

Hassan, S., Garmendia, L. \& Pavón, J. (2010). Introducing uncertainty into social simulation: Using fuzzy logic for agent-based modelling. International Journal of Reasoning-based Intelligent Systems, 2(2), 118-124

Hassan, S., Salgado, M. \& Pavón, J. (2011). Friendship dynamics: Modelling social relationships through a fuzzy agent-based simulation. Discrete Dynamics in Nature and Society, (p. 765640) 
Hellendoorn, H. \& Thomas, C. (1993). Defuzzification in fuzzy controllers. Journal of Intelligent \& Fuzzy Systems, $1(2), 109-123$

Izquierdo, L. R., Olaru, D., Izquierdo, S. S., Purchase, S. \& Soutar, G. N. (2015). Fuzzy logic for social simulation using NetLogo. Journal of Artificial Societies and Social Simulation, 18(4), 1

Jin, Y. (2003). Advanced Fuzzy Systems Design and Applications. Berlin/Heidelberg: Springer

Jones, H., Charnomordic, B., Dubois, D. \& Guillaume, S. (2009). Practical inference with systems of gradual implicative rules. IEEE Transactions on Fuzzy Systems, 17(1), 61-78

Klawonna, F. \& Novák, V. (1996). The relation between inference and interpolation in the framework of fuzzy systems. Fuzzy Sets and Systems, 81(3), 331-354

Klir, G. J. \& Yuan, B. (1995). Fuzzy Sets and Fuzzy Logic: Theory and Applications. Upper Saddle River, NJ: Prentice Hall PTR

Lee, K., Kim, S., Kim, C. O. \& Park, T. (2013). An agent-based competitive product diffusion model for the estimation and sensitivity analysis of social network structure and purchase time distribution. Journal of Artificial Societies and Social Simulation, 16(1), 3

Lee, K., Lee, H. \& Kim, C. O. (2014). Pricing and timing strategies for new product using agent-based simulation of behavioural consumers. Journal of Artificial Societies and Social Simulation, 17(2), 1

Mamdani, E. H. (1974). Application of fuzzy algorithms for control of simple dynamic plant. Proceedings of the Institution of Electrical Engineers, 121(12), 1585-1588

Mamdani, E. H. (1976). Advances in the linguistic synthesis of fuzzy controllers. International Journal of ManMachine Studies, 8(6), 669-678

Mamdani, E. H. (1977). Application of fuzzy logic to approximate reasoning using linguistic synthesis. IEEE Transactions on Computers, C-26(12), 1182-1191

Mamdani, E. H. (1994). Fuzzy control. A misconception of theory and application. IEEE Expert, 9(4), 27-28

Mamdani, E. H. \& Assilian, S. (1975). An experiment in linguistic synthesis with a fuzzy logic controller. International Journal of Man-Machine Studies, 7(1), 1-13

MATLAB (2016). Fuzzy logic toolbox user's guide. The MathWorks

Moraga, C. (2012). An essay on the interpretability of Mamdani systems. In P. P. Trillas, E andBonissone, L. Magdalena \& J. Kacprzyk (Eds.), Combining Experimentation and Theory, (pp. 61-72). Berlin/Heidelberg: Springer

Nait-Said, R., Zidani, F. \& Ouzraoui, N. (2008). Fuzzy risk graph model for determining safety integrity level. International Journal of Quality, Statistics, and Reliability, (p. Article ID 263895)

Neumann, M., Braun, A., Heinke, E.-M., Saqalli, M. \& Srbljinovic, A. (2011). Challenges in modelling social conflicts: Grappling with polysemy. Journal of Artificial Societies and Social Simulation, 14(3), 9

Novák, V. (1994). Fuzzy control from the logical point of view. Fuzzy Sets and Systems, 66(2), 159-173

Novák, V. (2012). Reasoning about mathematical fuzzy logic and its future. Fuzzy Sets and Systems, 192, 25-44

Olaru, D. \& Smith, B. (2005). Modelling behavioural rules for daily activity scheduling using fuzzy logic. Transportation, 32(4), 423-441

Pal, S. K. \& Mandal, D. P. (1991). Fuzzy logic and approximate reasoning: An overview. IETE Journal of Research, $37(5-6), 548-560$

Power, C. (2009). A spatial agent-based model of N-person prisoner's dilemma cooperation in a sociogeographic community. Journal of Artificial Societies and Social Simulation, 12(1), 8

Ragin, C. C. \& Pennings, P. (2005). Fuzzy sets and social research. Sociological Methods \& Research, 33(4), 423430

Ross, T. J. (2010). Fuzzy Logic with Engineering Applications (3 ${ }^{r d}$ Ed.). Chichester: Wiley 
Sabater, J., Paolucci, M. \& Conte, R. (2006). Repage: REPutation and imAGE among limited autonomous partners. Journal of Artificial Societies and Social Simulation, 9(2), 3

Shankar, S., Thangarasu, S. K., Mohanraj, T. \& Pravien, D. S. (2015). Prediction of cutting force in turning process: An experimental and fuzzy approach. Journal of Intelligent \& Fuzzy Systems, 28(4), 1785-1793

Smets, P. \& Magrez, P. (1987). Implication in fuzzy logic. International Journal of Approximate Reasoning, 1(4), 327-347

Smithson, M. (2012). Fuzzy set analysis for behavioral and social sciences. New York, NY: Springer

Stojić, G. (2012). Using fuzzy logic for evaluating the level of countries'(regions') economic development. $P a-$ noeconomicus, 59(3), 293-310

Thirumoorthi, P. \& Yadaiah, N. (2015). Design of current source hybrid power filter for harmonic current compensation. Simulation Modelling Practice and Theory, 52, 78-91

Valdés-Pasarón, S., Márquez, B. Y. \& Gaxiola, L. (2011). Knowledge and data engineering for analyzing the quality of education using fuzzy logic. In X. Wan (Ed.), Electrical Power Systems and Computers, (pp. 567-573). Berlin/Heidelberg: Springer

Van Leekwijck, W. \& Kerre, E. E. (1999). Defuzzification: Criteria and classification. Fuzzy Sets and Systems, 108(2), $159-178$

West, J. E. \& Linster, B. (2003). The evolution of fuzzy rules as strategies in two-player games. Southern Economic Journal, 69(3), 705-717

Wilensky, U. (1999). NetLogo. http://ccl.northwestern.edu/netlogo/. Center for Connected Learning and Computer-Based Modeling, Northwestern University. Evanston, IL

Yang, C.-L., Huang, Y.-C., Chang, Y.-K. \& Chu, C.-P. (2015). Fuzzy intelligent inference scheme for self-health estimation via blood pressure and body mass index in out-of-hospital. Journal of Intelligent \& Fuzzy Systems, 28(2), $775-786$

Zadeh, L. A. (1965). Fuzzy sets. Information and Control, 8(3), 338-353

Zimmermann, H.-J. (2001). Fuzzy Set Theory - and Its Applications (4 ${ }^{\text {th }}$ Ed.). Dordrecht: Springer Netherlands 